
January 1937

\title{
SATURATION BY WATER IN GAS ANALYSIS COMPENSATORS
}

By Joseph R. Branham

ABSTRACT

The effects of diffusion, convection, and evaporation of water from the glass surface in the compensators used in volumetric gas-analysis apparatus are discussed. The data presented show that serious errors result under certain common temperature conditions when, for the sake of thermal similarity, the compensator used is of the same shape as the bulbed burette.

\section{CONTENTS}

I. Introduction $\quad 59$

II. Evaporation from glass surfaces $\ldots \ldots \ldots$

III. Convection

IV. Diffusion

V. Apparatus and procedure

VI. Experimental data

VII. Discussion of results _.

VIII. Conclusion

\section{INTRODUCTION}

In volumetric gas analysis, over mercury, measurements are usually made by observing the position of the mercury meniscus in the burette in which the pressure of the gas has been adjusted by connecting through a manometer to a "compensator." In order that the gas in the compensator and burette may be under similar thermal conditions, the compensator is usually of the same shape and size as the burette and stands beside it in a water jacket. Unless the gas is dried before each measurement, a rare procedure, a part of its pressure is exerted by water vapor. To make this pressure definite and to automatically correct for it, a little liquid water is kept in the burette and compensator to insure a saturation pressure of water vapor in both. Under these conditions each unit volume of the burette will contain the same fraction of a mole of gas, excepting water vapor, throughout the analysis, and the volumes measured may be added to or subtracted from each other without correction.

Burettes for special purposes are frequently used for the analyses of gases the percentage composition of which varies but slightly from some normal condition. Such burettes ordinarily consist of one or more bulbs of relatively large diameter connected by graduated tubing of small diameter, so proportioned that the significant measurements are always read in one of the small calibrated tubes. 
In the apparatus used by Carpenter ${ }^{1}$ for the analysis of air the burette and compensator each consists of two large bulbs connected by three lengths of capillary tubing $2 \mathrm{~mm}$ in diameter. The vertical distance between the calibration marks permits readings of 0.001 percent of the burette volume to be made with reasonable precision. A corresponding precision with a cylindrical burette would be only 0.02 percent of the volume.

Recently the author undertook a series of analyses of outdoor air by means of the Carpenter apparatus. Some of the results were discouraging, and the possibility that the rate of saturation of the compensator was insufficient seemed the most probable explanation. It is customary to put water into the compensator of this apparatus but once during the life of the apparatus unless accidents occur or the water becomes dirty after several years of use. This water collects in the bottom of the compensator, and water vapor from this source must pass through $48 \mathrm{~cm}$ of capillary tubing in order to reach the upper bulb of the compensator. The manipulation of the apparatus requires the frequent admission of laboratory air, which may be relatively dry, to the top of the compensator, and the gas confined in this part is in contact through capillary connections with a 20 -percent solution of potassium hydroxide which serves as the manometric fluid.

In the course of an analysis some transfer of gas back and forth between the compensator and manometer is unavoidable. The admission of laboratory air and the mild desiccating action of the potassium hydroxide solution may be expected gradually to exhaust the film of visible water on the compensator walls until only an adsorbed film of water is available to saturate the gas in the compensator. The thickness of this film will not necessarily be uniform and will depend on the recent history of the apparatus. One part of the film may be in equilibrium with the water vapor from the bottom of the compensator, while another part may be in equilibrium with the water vapor from the potassium hydroxide or the partial pressure of the laboratory air last admitted to the compensator.

\section{EVAPORATION FROM GLASS SURFACES}

The vapor pressure of films of water adsorbed on glass has been measured by McHaffie and Lenher. ${ }^{2}$ According to these authors, the vapor pressure of the film is a function of its thickness. Their data indicate that there should be a significant difference between the vapor pressure of the visible film in the burette and the adsorbed film in the compensator whenever the latter film is less than several hundred molecules thick.

If a cylindrical compensator, $1 \mathrm{~cm}$ in diameter, is saturated at $19^{\circ} \mathrm{C}$, approximately 60 molecular layers of water must evaporate from its walls to saturate the gas in it at $20^{\circ} \mathrm{C}$. Within certain temperature limits, depending upon the history of the apparatus, sufficient water will evaporate from the film on the compensator walls to saturate the gas in the compensator. Above this limit water vapor must travel up the tube from the lower reservoir if the gas is to become saturated.

1 Thorne M. Carpenter, J. Metabolic Research 4, 1-25 (1923).

${ }^{2}$ I. R. McHaffie and S. Lenher, J. Chem. Soc. 127, 1559 (1925). 


\section{CONVECTION}

The movement of water vapor in tubes of very small internal diameter is chiefly by diffusion. Tubes of 6 and $9 \mathrm{~mm}$ diameter were used by different observers to determine the value of the diffusion constant of water vapor into air. ${ }^{3}$ The data obtained by these investigators are in fair agreement and indicate that the effects of convection were small if present at all, under the conditions of their experiments.

Data on the conduction of heat across air spaces may be pertinent to the discussion of the movement of water vapor in so far as density relations are involved in the two cases. Such data indicate that convection becomes increasingly important in the conduction of heat as the distance across the air space increases and that convection is negligible, if it occurs at all, when the distance across the space is less than $1 \mathrm{~cm}^{4}$

As a supplement to the data presented in this paper, the times required for ammonia vapor to pass up vertical tubes of different diameters were measured. Glass tubes $57 \mathrm{~cm}$ long and 3.1, 3.8, $7.6,8.8,15.4,19.0$, and $24.3 \mathrm{~mm}$ in diameter were used. Strips of congo red paper (blue by treatment with $\mathrm{HCl}$ gas) were held in place by cotton plugs at the upper ends of the tubes. The lower ends were immersed simultaneously in a solution of ammonium hydroxide and the times required for sufficient ammonia to pass up the tubes and begin to change the color of the indicator were measured. The relative times (time in the largest tube being taken as the unit) as calculated from the average of seven experiments, in the order of the diameters given above, were $3.3,3.3,3.1,2.9,2.6,2.0$, and 1 . In the individual experiments the relative times were much larger when concentrated solutions of ammonium hydroxide were used and approached unity with dilute solutions which indicates that the density gradient in the tubes as well as their diameters is a controlling factor in initiating convection currents.

\section{DIFFUSION}

The rate of diffusion of water vapor into air partially saturated with water vapor may be calculated closely enough to permit a conclusion to be drawn as to the order of magnitude of the time required to reach any given degree of saturation in a cylindrical vessel. The general equation for diffusion (Int. Critical Tables V, 62)

$$
\frac{\delta p}{\delta t}=D \frac{\delta^{2} p}{\delta x^{2}}
$$

was solved and expanded ${ }^{5}$ into a form that can be applied to the diffusion of water vapor occurring in narrow cylindrical compensators. The calculations were made for tubes of various lengths, and the time required to reach a given degree of saturation was plotted against the

\footnotetext{
${ }^{3}$ A. Winkelmann, Ann. Physik 258, 7 (1884).

M. Le Blane and G. Wuppermann, Z. phys. Chem. 91, 150 (1916)

4 H. C. Dickinson and M. S. Van Dusen, Am. Soc. Refrigerating Engrs. J. 3, (1916).

P. E. Palmer and E. R. Weaver, BS Tech. Pap. 18, 35 (1924) T249.

White, Phys. Rev. 10, 743 (1917).

R. B. Kennard, BS J. Research, 8, 787 (1932) RP452.

5 The author is indebted to C. S. Cragoe and C. Snow of this Bureau for the mathematical treatment of the diffusion data.
} 
degree of saturation. The data are presented in figure 1. The calculations assumed that the compensators were saturated at $20^{\circ}$ and the temperature was then changed to and held constant at $21^{\circ}$. Gravitational effects were neglected and uniform temperatures were assumed throughout the system. The difference between the average partial pressure in the tube, at time $t$, and the saturation pressure of water at $21^{\circ}$ is the ordinate, and time is the abscissa. The data indicate that approximately 5 hours must elapse in order that the volume readings from an apparatus employing a 48-cm cylindrical compensator will be accurate within $0.01 \mathrm{~mm}$ of $\mathrm{Hg}$ or approximately

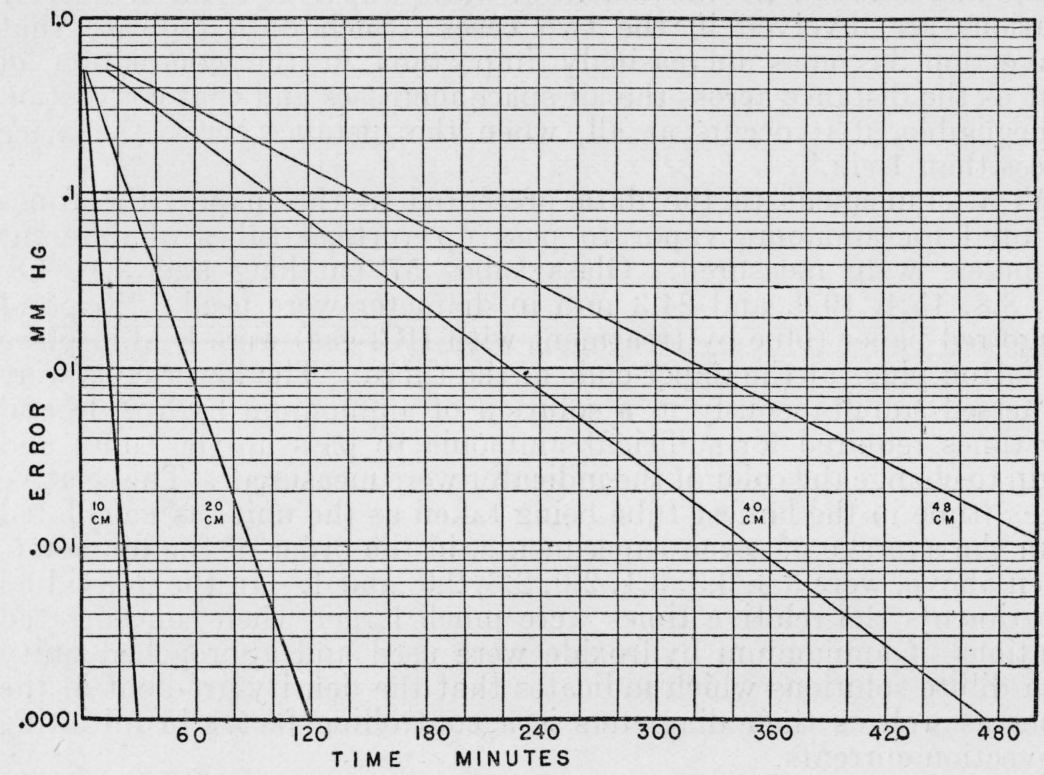

Figure 1.-Calculated deficiency of saturation by diffusion after a $1^{\circ}$ change of temperature.

0.001 percent. When the compensator employed is of the bulb type and the bulbs comprise over 90 percent of the total volume, the time required to reach a given degree of saturation will be much longer than that indicated for cylindrical compensators. It is interesting to note that the time required to reach any given degree of saturation is proportional to the square of the length of the compensator, so that better compensation may be obtained by making this part of the apparatus shorter than the burette, even at the sacrifice of a certain degree of thermal similarity between the burette and compensator. The experimental data to be presented indicate that the same purpose may be accomplished by using a full-length compensator $1.5 \mathrm{~cm}$ or more in diameter.

\section{APPARATUS AND PROCEDURE}

The experimental data were obtained by means of apparatus $A$ and $B$, shown in figure 2. The right side of $A$ is similar to the compensator of the Carpenter apparatus. The right side of $B$ is of the 
same shape as the compensator of the gas-analysis apparatus used for general purposes. In the remainder of this paper the terms "burette" and "compensator" will be used to signify respectively the left and right sides of the apparatus. The diameter of the capillaries is $2 \mathrm{~mm}$, and the diameter of the cylinder about $15 \mathrm{~mm}$. Apparatus $B$ was made by substituting the cylinder for the bulbed compensator after the data had been obtained from $A$. The left side of the apparatus, which was common to both $A$ and $B$, was etched inside so that a film of liquid would be retained on the surface and its presence could be observed. The presence of this film at all times during the experiments corresponds to the condition which the analyst endeavors to maintain in a burette, and the pressure changes occurring in this side with changes of temperature may be regarded as similar to those occurring in a burette during an analysis.

The following procedure was employed in preparing the apparatus, except as noted later for the sixth experiment. Both sides of the apparatus were cleaned with a solution of potassium dichromate in concentrated sulphuric acid, washed with distilled water and dried by a current of room air. Room air bubbled through water at $24^{\circ} \mathrm{C}$ was passed for an hour through the right-hand side of each apparatus. The left side of the apparatus was then filled with water which was allowed to drain out slowly until the amount left was just sufficient to serve as a manometric fluid. The stopcocks were then turned to connect the burette and compensator of the ap-

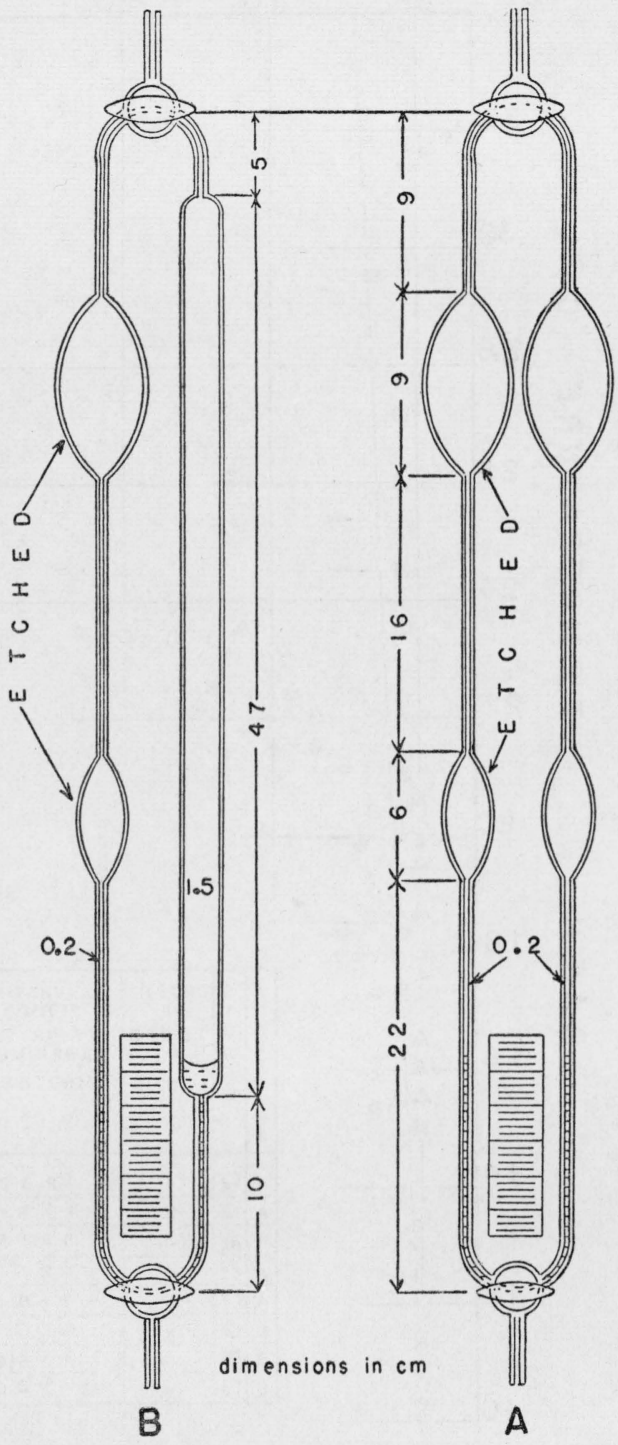

Figdre 2.-Apparatus used to measure the difference of pressure between the burette and compensators. paratus, and part of the water in the burette ran into the lower part of the compensator and served as a manometric fluid and as a saturating reservoir. The upper stopcock was then closed and the apparatus $110639-36-5$ 
completely immersed in a vigorously stirred water bath. In addition to the stirring, the apparatus was rotated by hand about its vertical axis before observations were made, in order to obtain

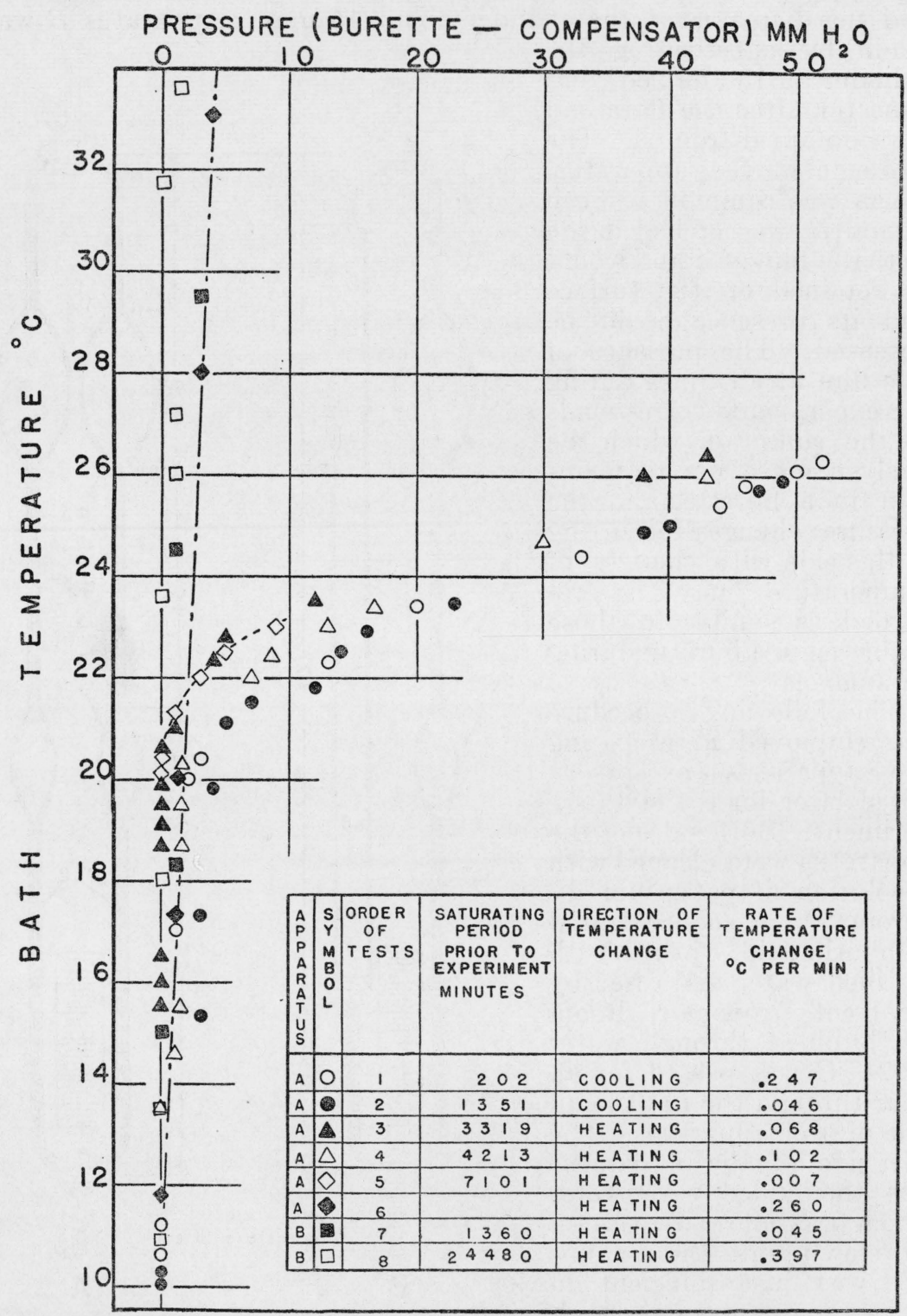

Figure 3.-Effect of change of temperature on the difference of pressure between the burette and the compensators.

similar thermal conditions in both sides. Rapid changes of temperature were accomplished by the addition of ice or warm water to the bath; gradual changes were accomplished by means of a copper U-tube immersed in the bath and connected to the hot-and cold-water supply. 


\section{EXPERIMENTAL DATA}

The experimental data are presented in figure 3. On this graph the temperature is plotted as the ordinate against the difference of pressure between the two sides of the apparatus as the abscissa. ${ }^{6}$ The table at the bottom contains most of the data pertinent to the experimental procedure. The saturating period indicated is the total time that the gas in the compensator had been in contact with the manometric fluid.

The scattering of the points may be attributed chiefly to the behavior of the manometric system. The nature of the work necessitated the use of water as the manometric fluid, and there was a greased stopcock at the bottom of the manometer. The precision of the experiments does not permit a conclusion to be drawn concerning the exact degree of compensation obtained by the two compensators under the conditions of the experiments. The information obtained was a qualitative rather than a quantitative measure of the saturation of the compensators and of the role played in this process by diffusion, convection, and surface evaporation.

\section{DISCUSSION OF RESULTS}

Previous to the sixth experiment the apparatus was filled with water, which was allowed to drain leaving the inner surfaces of both sides of the apparatus visibly wet. Under these conditions only a slight difference of pressure existed between the two sides of the apparatus over the entire temperature range investigated, in spite of the very rapid change of temperature. The slight difference in the degree of saturation may indicate the effect of the etched surface or a small thermal difference between the two sides of the apparatus. There is no indication of a sharp change in the degree of saturation reached in the two sides of the apparatus.

The first five experiments, $A 1$ to $A 5$, inclusive, were made without altering the stopcocks and only the left side of the apparatus was visibly wet. The right side had been subjected to the preliminary treatment described in section $V$.

The plotted data from these five experiments indicate an increasing difference in the degree of saturation between the two sides of the apparatus at temperatures above $20.5^{\circ} \mathrm{C}$. Below this temperature the compensation was about as good as when both sides of the apparatus were visibly wet. The break in the curve is sharp and the temperature at which it occurs is nearly independent of the rate of temperature change or of the period of saturation prior to the experiment. There is a slight difference between the observations when the apparatus was being heated and those when it was being cooled. This difference may be an indication of inaccuracy in the manometric system. It is evident that neither diffusion nor convection supplies water vapor to the compensator, above $20.5^{\circ} \mathrm{C}$, at rates which are adequate to saturate it when the rate of change of temperature is $0.007^{\circ}$ per minute.

\footnotetext{
6 This method of presenting the data differs from a gas analysis determination in that the volume of the compensator is not constant but changes with the movement of the manometric fuid. If the volume of the compensator in the experimental apparatus had been kept constant, the pressure differences given in figure 3 would have been about 50 percent greater than those recorded.
} 
The slope of the curves above $20.5^{\circ} \mathrm{C}$ in the first five experiments is determined by the amount of water vapor supplied to the compensator by evaporation from the walls, and by diffusion, and convection of water vapor from the water meniscus during the experiment. Within the limit of experimental error these slopes are the same and indicate that equal amounts of vapor were supplied in all five experiments. Since the saturating period per degree change of temperature (the reciprocal of the rate of temperature change) varied from 4 minutes in the first to 143 minutes in the fifth experiment and did not measurably affect the amount of vapor supplied, the effects of convection and diffusion must have been very small. In view of this fact the saturation attained when heating below $20.5^{\circ} \mathrm{C}$ must have been affected by the evaporation of the water on the walls of the compensator.

The experiments $B 7$ and $B 8$ were made with apparatus $B$, figure 2 . The cylinder which replaced the bulbed compensator was subjected to the same preliminary treatment as the latter and presumably had a similar film of adsorbed water on its inner surface. The data indicate that reasonably accurate compensation was afforded by the cylindrical compensator at higher rates of change of temperature than will be encountered under normal laboratory conditions, and at temperatures above those at which the bulbed compensator may fail to give even approximately accurate results.

On the basis of the calculated rates of diffusion given in figure 1 , the saturation of the cylindrical compensator resulted chiefly from convection. The rapid equalization of pressure in the two sides of apparatus $B$, as shown by the movement of the manometric fluid when this apparatus was subjected to sudden increases of temperature, and by the absence of a similar movement under like conditions with apparatus $A$, also indicates that convection was chiefly responsible for the saturation of apparatus $B$ at the higher temperatures. The data on the movement of ammonia in tubes of different diameters also indicate that the effects of convection will be greater in the wide cylindrical compensator.

The accuracy of volumetric measurements in a gas-analysis apparatus will be a function of the time required to saturate the compensator as compared with the saturation period of the burette. The presence of water on the walls of the burette ensures rapid saturation at all times and the accuracy of the apparatus, insofar as saturation is involved, will depend on the time required to saturate the compensator and the rate at which the temperature of the apparatus is increasing.

The degree of saturation of a "dry-walled" compensator must necessarily lag behind that of a "wet-walled" burette when the temperature of the apparatus is increasing, and the difference in saturation will be a function of the rate of temperature change as well as the shape of the compensator. This difference in saturation may be made small by eliminating any features in the design of the compensator which hinder the free movement of water vapor, but cannot be eliminated entirely if the distance that water vapor must travel is different in the two vessels being saturated. 


\section{CONCLUSION}

In order to obtain a similar rate of transfer of heat from a water jacket through the walls of the burette and compensator of a gasanalysis apparatus, these parts are made of the same material and are of the same shape and size, but unavoidably are not of the same thickness. The benefits in volumetric accuracy resulting from this thermal similarity must be very small because the burette and compensator stand close together in a water jacket and the position of the mercury in the burette may be anywhere between the top and bottom of the water jacket, while the entire length of the compensator is always gas-filled.

To maintain a saturated condition of the gas, the water in a compensator of the type used by Carpenter is confined in the bottom of a tube of $2 \mathrm{~mm}$ diameter, and visible water on the walls above this tube is avoided because it tends to run down and plug the narrow tubes joining the compensator bulbs.

The data indicate that the rate of travel of water vapor upward from the bottom of a capillary $2 \mathrm{~mm}$ in diameter is too slow to appreciably affect the degree of saturation of a gas confined in the body of a bulbed compensator. If there is no visible water on the walls of such a compensator, the only source of water vapor, for saturation purposes, is the film of adsorbed water on the glass walls. The thickness of this film and its vapor pressure will depend on the temperature of the compensator and the amount of water vapor in the gas in the compensator. Above some limiting temperature, corresponding to the break in the curve of figure 3, which will depend on the volume and humidity of the laboratory air admitted to the compensator, the manipulation of the apparatus and the vapor pressure of the potassium hydroxide solution in the manometer, the gas in the compensator will not be saturated; and serious volumetric errors will occur in an analysis whenever the temperature of the apparatus changes in either direction. At temperatures which are lower than the temperature which is critical for the particular conditions and apparatus, the error of compensation will be small and will depend on the relative rates of heat transfer to the burette and compensator rather than on the travel of water vapor. The data of figure 3 indicate that errors resulting from incomplete saturation in the compensator may be largely avoided by using a relatively wide cylinder as a compensator. This, of course, will result in the sacrifice of whatever benefits there may be in the thermal similarity of the burette and compensator. One method to retain partial thermal similarity, and at the same time insure more rapid saturation of the gas in the bulbs of the compensator, would be to extend the capillary tubes up into the body of each of the bulbs, so that the walls of the bulbs could be visibly wet without danger of the water in them draining into the capillaries to form slugs.

Washington, September 4, 1936. 